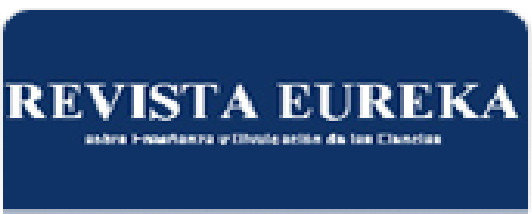

Córdova-Frunz, José Luis; Vargas-Fosada, Rubicelia; Viniegra-Ramírez, Margarita

Revista Eureka sobre Enseñanza y Divulgación de las Ciencias

E-ISSN: 1697-011X

revista@apac-eureka.org

Asociación de Profesores Amigos de la

Ciencia: EUREKA

\section{España} Revista Eureka sobre Enseñanza y Divulgación de las Ciencias, vol. 10, núm. 2, abril, 2013, pp. 211 -

221

Asociación de Profesores Amigos de la Ciencia: EUREKA

Cádiz, España

Disponible en: http://www.redalyc.org/articulo.oa?id=92026042009

Cómo citar el artículo

Número completo

- Más información del artículo

- Página de la revista en redalyc.org 


\title{
Traducción del lenguaje cotidiano al gráfico: una experiencia de fábula
}

\author{
José Luis Córdova-Frunz, Rubicelia Vargas-Fosada, Margarita Viniegra-Ramírez \\ Depto.de Quimica. Universidad Autónoma Metropolitana.México.joseluiscordovafrunz@gmail.com
}

[Recibido en febrero de 2012, aceptado en diciembre de 2012]

\begin{abstract}
La resolución de problemas implica traducir lenguajes, por lo que frecuentemente se analizan los problemas sobre la traducción del lenguaje cotidiano al algebraico. Se ha investigado poco acerca de la traducción entre los lenguajes cotidiano y gráfico, siendo que esta traducción obliga al uso de habilidades superiores de pensamiento: selección de información, de variables relevantes, de escalas, etc. Presentamos un estudio exploratorio en este tema, de las habilidades de estudiantes universitarios de reciente ingreso con el fin de evaluar el nivel de desarrollo de estas destrezas. Hacemos uso de un ejercicio no estructurado en el que los alumnos deben graficar la información que brinda un texto. Se empleó una lista de cotejo para el análisis de resultados; éstos muestran que los alumnos tienen poca habilidad para la traducción del español al lenguaje gráfico y tienden a confundir una gráfica con una trayectoria.
\end{abstract}

Palabras clave: Habilidades superiores; traducción lenguajes; lenguaje gráfico.

\section{Dayly language to graphic translation: a fable experience}

Problem solving involves translating languages; the translation between daily and algebraic languages has received more attention than the translation between daily and graphic languages, even though the later requires the use of higher thinking skills like selection of the information, of the relevant variables, scales, etc. We present here an exploratory study in this item, around the skills of freshman students in the university. We employ a nonstructured problem in which the students have to graph the information given in a text. We used a check list to analyse the results; these show that students have poor skills for the translation between Spanish and graphic language and they have a tendency to confuse a graph with a trajectory.

Key words: Higher thinking skills; language translation; graphic language.

\section{Introducción}

La resolución de problemas es una de las estrategias más usadas en la enseñanza-aprendizaje de las ciencias. El resolver problemas desarrolla la capacidad de abstracción, análisis y síntesis, operaciones necesarias para el aprendizaje.

El primer paso del método propuesto por Polya (1989) es comprender el problema. Esta comprensión implica la selección de la información importante para trazar un plan de solución, así como nombrar a los conceptos involucrados en la actividad. En lo que se refiere al empleo de literales para la identificación de datos e incógnitas, los alumnos prefieren usar números en vez de símbolos $\mathrm{y}$, generalmente, lo hacen sin utilizar las unidades correspondientes. Estas dificultades están directamente relacionadas con la traducción de los lenguajes: algebraico, cotidiano y gráfico. Newton (1707) en su manual de álgebra escribió «Para resolver un problema referente a números o relaciones abstractas de cantidades basta con traducirlo, del inglés u otra lengua al idioma algebraico».

En nuestra experiencia en el aula, la traducción al lenguaje algebraico o gráfico es una de las principales limitaciones a las que se enfrentan los estudiantes del nivel superior. Las deficiencias parten desde la comprensión del lenguaje cotidiano (LC), por ejemplo doble de, cuadrado de, proporción, etc. pues no pueden elaborar enunciados gramaticales equivalentes. Cabe aquí una reflexión sobre la ambigüedad asociada al LC, por ejemplo decir "yo gano dos veces más que tú" es menos preciso que "yo gano el doble que tú" o que "yo gano dos veces 
lo que tú ganas". Si traducimos al lenguaje algebraico, el primer enunciado podría ser $y=2 x \mathrm{o}$ $y=2 x+x$ donde $y$ es los que yo gano y $x$ lo que tú ganas.

Para ejemplificar la dificultad en la traducción al lenguaje algebraico proporcionamos los ejemplos de la tabla 1 obtenidos de tareas de alumnos de reciente ingreso a la universidad.

Tabla 1. Ejemplos de traducción al lenguaje algebraico elaborados por alumnos.

\begin{tabular}{|l|c|}
\hline \multicolumn{1}{|c|}{ Enunciado } & Traducción elaborada por los alumnos \\
\hline $\begin{array}{l}\text { 1. El volumen de un cubo es igual a la } \\
\text { longitud de la arista elevada al cubo }\end{array}$ & $V^{3}=\log (a)^{3}$ \\
\hline 2. El número de Avogadro es & $6.02^{23}$ \\
\hline 3. Cinco veces $R$ & $R^{5}$ \\
\hline 4. El doble de la suma de $x$ y 3 & $2 x+3$ \\
\hline
\end{tabular}

En el enunciado 1 de la Tabla 1, la traducción es en el mismo orden de lectura, además está elaborada de manera mecánica, es decir término a término: el volumen de un cubo es $V^{3}$ y la longitud es log. En el 2, cuando decimos el número de Avogadro es 6.02 por 10 a la 23, el alumno no nota la diferencia entre la notación de la calculadora y la notación científica. En el ejemplo 3, existe una ambigüedad: la palabra "veces" oculta si la $R$ se usa como factor o como sumando. El ejemplo 4 muestra que los paréntesis son irrelevantes para los alumnos.

En los ejemplos anteriores, hay interpretaciones resultantes de generalizaciones en principio válidas, pero que no consideran las inevitables limitaciones de toda generalización y que las representaciones siguen los principios de economía y contexto. Los enunciados útiles en la vida cotidiana carecen de la precisión y concisión de los enunciados matemáticos cuya sintaxis es más rigurosa y cuya semántica es flexible; los enunciados cotidianos tienen, además, un nivel de redundancia del que carecen los matemáticos. Si en un caso predomina la eficacia, en el otro es el rigor. No es sólo que los signos (semántica) tengan diferente universo conceptual es, además, que la construcción de los enunciados matemáticos (sintaxis) es completamente distinta (posición-prioridad, posición-operación, posición-agrupamiento, etcétera), por ejemplo la lectura de los enunciados gramaticales es de izquierda a derecha, mientras que la de un enunciado matemático es del paréntesis interior al exterior.

Hemos notado que existe mayor trabajo hecho respecto a la traducción de LC al algebraico (Flores-Martínez y Peñas-Troyano, 2003) que del LC al gráfico; de hecho, el National Council of Teachers of Mathematics (NCTM 2011) no hace mención explícita de ésta.

El objetivo de este trabajo es analizar las habilidades de los alumnos universitarios de primer año sobre la traducción de la información cualitativa presentada en LC al gráfico, mediante un ejercicio no estructurado. Es importante enfatizar que hemos usado un ejercicio no estructurado ya que en la educación escolar básica y media, los alumnos aprenden a resolver problemas con:

- datos e incógnitas explícitos,

- una solución única producto de una secuencia definida, generalmente lineal,

- procedimientos que son una repetición de lo mostrado en la clase.

Los problemas que se ajustan a la lista anterior, los llamaremos ejercicios estructurados (como los de final de capítulo de libro de texto o los libros de la serie Schaum). Si bien esta estrategia es adecuada en el proceso de enseñanza aprendizaje, ya que obliga a la necesaria mecanización 
de los procedimientos, los ejercicios no estructurados tienen la ventaja de que estimulan el desarrollo de otras habilidades requeridas para la resolución de problemas como pueden ser: identificación de variables, traducción entre lenguajes, discriminación de información, revisión de la solución, etcétera.

\section{Metodología}

Se adaptó la fábula de Esopo de la "Liebre y la tortuga" (Esopo, 2011) como el ejercicio no estructurado, para que los alumnos elaboraran gráficas de la carrera descrita en esta fábula. Este ejercicio nos permitirá conocer las habilidades de los alumnos en este sentido, esto es, sabemos que los alumnos pueden hacer una gráfica de una tabla de datos numéricos, pero no es la habilidad de graficar lo que queremos analizar sino todo el proceso involucrado en la traducción de lenguajes: selección de la información, representación cualitativa, versión cuantitativa de la anterior, la definición del dominio de las variables y la elaboración de la gráfica.

La muestra consistió en 118 alumnos cuyas edades fluctúan entre los 18 y 20 años. Todos ellos cursaban, en el momento del estudio, el primer año de una licenciatura en ciencias básicas o ingeniería. La mayoría de estos alumnos provienen del sistema de educación pública del país y son hijos de padres que no son profesionistas (Rodríguez-Lagunas, 2009).

Se repartió a dos grupos de 25 alumnos cada uno la fábula de Esopo, versión 1, para que resolvieran las actividades en una clase de 60 minutos. Se les explicó que el ejercicio no tendría repercusiones en su calificación. Los resultados esperados eran dos gráficas: una gráfica de distancia contra tiempo y otra de velocidad contra tiempo, para los dos animales.

La revisión global de estos resultados llevó a una lista de cotejo preliminar. Su aplicación detallada obligó a precisar los siguientes puntos a fin de evitar la dispersión de respuestas, para una segunda aplicación:

- Con el fin de acotar las gráficas añadimos el dato numérico de la distancia metaorigen y la duración de la carrera.

- Se proporcionaron gráficas con los ejes ya etiquetados con las variables correspondientes y se solicitó a los alumnos que incluyeran las unidades.

- Se subrayó la frase "velocidad constante".

La versión 2 y su respectiva lista de cotejo se encuentran en el Anexo 1. Todos los cambios se hicieron en la sección Actividades del documento entregado a los alumnos; el texto de la fábula permaneció igual en las dos versiones. Como mencionamos, estos cambios resultaron tanto de haber aplicado una lista de cotejo insuficiente, así como de resultados no evaluables, por ejemplo la omisión por parte de los alumnos de etiquetas en los ejes impidió interpretar la traducción de los alumnos en sus gráficas. Lo mismo llevó al rediseño de la lista de cotejo.

La versión 2 de las actividades se aplicó en clase de 60 minutos a 68 alumnos de primer año de los cuales 30 ya habían cursado Mecánica y Fluidos (primer curso de física en la universidad) que incluye nociones de cinemática. Para hacer el análisis de resultados la lista de cotejo se diseñó para contabilizar exclusivamente las respuestas correctas. También se calculó el porcentaje de aciertos por alumno para hacer un análisis del desempeño general del grupo. Los resultados de la aplicación de ambas versiones se discuten en el presente trabajo. 


\section{Resultados y discusión}

En la aplicación de ambas versiones de la actividad la mayoría de los alumnos terminó el ejercicio en el tiempo establecido (1 hora). Ningún alumno se negó a hacer el ejercicio, y se mostraron participativos a sabiendas que no contaba para su calificación. Durante el ejercicio los alumnos no hicieron preguntas de ningún tipo y detectamos que algunos copiaban. Es importante resaltar que cuando se trata de exámenes que son parte de la calificación sí es frecuente que los alumnos pregunten sobre lo que no entienden y, sobra decirlo, copien.

En cuanto a los supuestos indispensables para la resolución del ejercicio en la versión 1 son:

- Fijar una meta en el eje de distancias.

- Duración de la carrera.

- Las velocidades de la liebre y la tortuga.

- Aceleraciones infinitas para la liebre.

- Tiempo de partida de la liebre.

- Duración de los descansos de la liebre.

Dado que en la primera versión aplicada los alumnos no hicieron los supuestos 1 y 2 , los resultados fueron imposibles de interpretar (los pocos que pusieron escalas, 10 de 42, implicaban velocidades irreales o carreras de 2 metros, la mayoría no indicaban títulos de los ejes). En la segunda versión (véase Apéndice) les asignamos valores numéricos tanto a la distancia como a la duración de la carrera, con lo que sólo tendrían que suponer, para la liebre, la velocidad, el tiempo de partida y la duración de sus descansos. Estos cambios llevaron también a cambiar la lista de cotejo. En lo que sigue, hablaremos sólo de los resultados de la versión 2 cuya lista de cotejo contiene 22 ítems, los 12 primeros para la gráfica distancia contra tiempo, y los siguientes 10 para la gráfica velocidad contra tiempo.

Los 22 puntos de la lista de cotejo permiten calcular el porcentaje de aciertos de cada alumno; la figura 1 presenta la distribución de aciertos por alumno. Esta gráfica nos muestra que casi el $87 \%$ de los alumnos obtuvo menos del 50\% de aciertos, y de éstos, aproximadamente la tercera parte sólo logró el 30\% de aciertos. Ningún alumno contestó acertadamente a todos las preguntas y solamente $0.3 \%$ de alumnos lograron más del $70 \%$ de respuestas correctas.

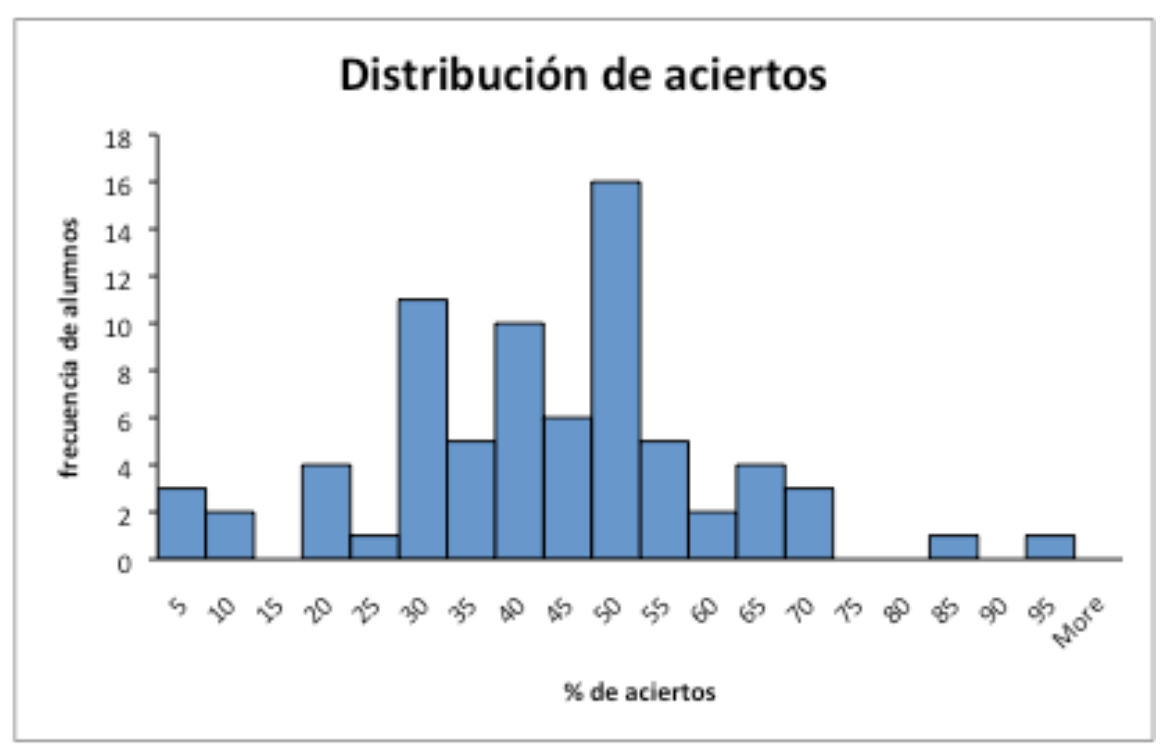

Figura 1. Distribución de aciertos, segunda versión. 
En cuanto al análisis de aciertos por ítem, de acuerdo a la gráfica 2 y considerando solamente los 12 primeros ítems de la lista de cotejo (gráfica $d v$ s $t$ ) se ve menor frecuencia de aciertos en los ítems 4, 5, 6 y 11 que corresponden a las preguntas:

4. La liebre parte después de la tortuga

5. Explicita los supuestos

6. Los supuestos son correctos

11. Se notan los rebases de la liebre

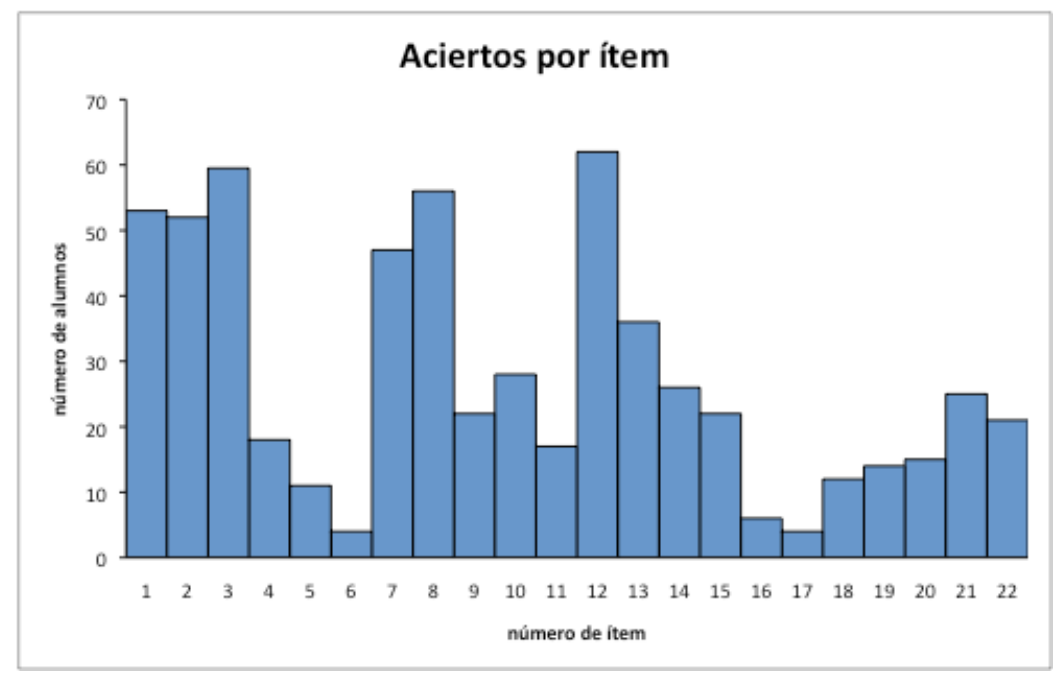

Figura 2. Alumnos que responden correctamente a los ítems.

Respecto al ítem 4, es importante notar que los enunciados: "confiada en su ligereza, la liebre dejó partir a la tortuga y se quedó remoloneando...." "luego, empezó a correr..." emplean una metáfora y un término no cotidiano, y puede ser la razón para que, en la gráfica de la liebre, no se exprese que ésta parte tiempo después que la tortuga. Esto es, la información para contestar adecuadamente a este ítem se encuentra en dos enunciados no cotidianos, además, en párrafos diferentes. La comprensión de la lectura es por bloques (Sanfelices, 1990) y una o dos palabras oscuras cancelan la información de ese bloque. Como menciona Gutiérrez Rodilla (1998), el contenido sugiere al lector los esquemas y estrategias de interpretación.

Los ítems 5 y 6 indican que la mayoría no explicita supuestos aunque los utilizan, y los supuestos que expresan son en general incorrectos o bien no son "supuestos": son información explícita en el enunciado. Una de las posibles razones de lo anterior es que en los problemas de clase generalmente los supuestos no se hacen explícitos por parte de los profesores; la atención está centrada en el resultado y el procedimiento, y no en lo que rodea al problema, por ejemplo: la verificación, identificación de supuestos, cómo influye su modificación, la confirmación del resultado por otras vías como trabajo en reversa o por análisis dimensional. Muchas de estas actividades no se realizan en el aula por lo que queda trunco el trabajo, en otras palabras, los profesores no promueven las habilidades fundamentales para la resolución de problemas. Por otro lado, explicitar un supuesto implica la toma de decisiones, la vida urbana no facilita la toma de decisiones, o las disfraza o las evita. El bombardeo de instrucciones de la prensa y los medios: compre, gaste, vaya, publique, etc. invita a que la rutina domine las acciones. Se actúa, pero poco se decide. Feldenkrais (1981) afirma que "nos esforzamos demasiado en obrar correctamente" y no atendemos a la forma en que hacemos las cosas rutinarias, "si prestamos atención a nuestro modo de hacer las cosas, 
tal vez descubramos otras maneras de hacerlas; tal vez tengamos la posibilidad de elegir (op.cit).

Los ítems 10 y 11 están relacionados por un cambio de pendiente en la gráfica de distancia contra tiempo, puesto que el 10 se refiere a los descansos de la liebre y el 11 a los rebases. Es importante mencionar que algunos alumnos representaron los descansos de la liebre como puntos grandes sobre la recta o como discontinuidades de la misma (en la gráfica $d v s$ t 18 lo hacen y en la de $v$ vs $t$ son 12). Otro aspecto a resaltar es que 48 alumnos consideran una velocidad menor o igual para la liebre, por lo que las pendientes dibujadas impiden que haya rebases. Al parecer identifican la gráfica con el recorrido como si ésta fuera un mapa. Es importante señalar que en los libros de texto de física de la educación media superior, se acostumbra representar gráficas de posición contra tiempo, por ejemplo para el movimiento rectilíneo uniforme; ilustramos lo anterior en las Figura 3 y 4 tomadas de libros de física elemental (Arciniega y Jaime, 2011; Griffith, 2008). En la primera imagen se grafica la distancia versus el tiempo para el movimiento rectilíneo uniforme, sin embargo el texto que precede a la figura habla de graficar las posiciones, por lo que no se distinguen los conceptos de posición y distancia al origen. En la Figura 4, correspondiente a tiro parabólico, no se etiquetan los ejes coordenados y se hace imposible la distinción entre posición y distancia recorrida, más aún, se coloca la imagen del lanzador en el origen de la gráfica. Estas imágenes ejemplifican los orígenes de la confusión en los alumnos de conceptos tan distintos como posición, distancia y tiempo.

Vamos a graficar ahora las posiciones para cada tiempo en un diagrama de distancia tiempo.

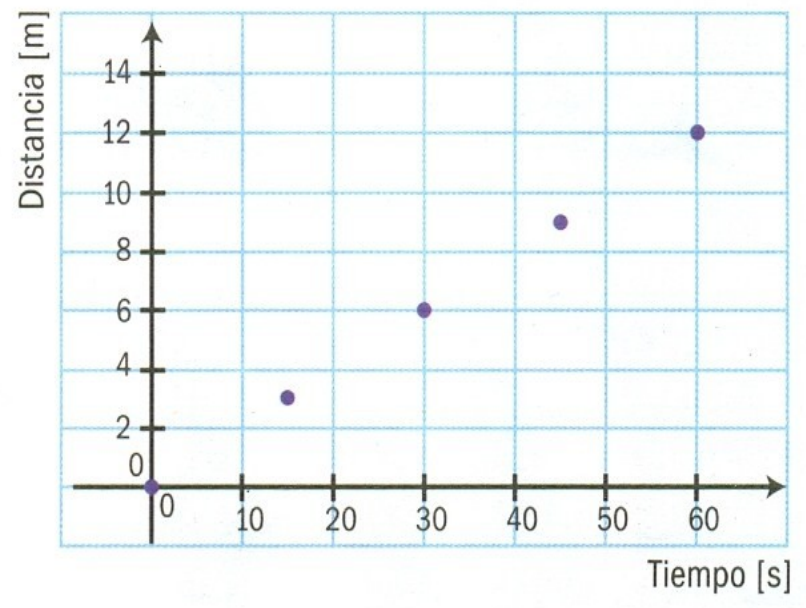

Imagen 2.15. Diagrama de distancia vs. tiempo.

Figura 3. Diagrama de distancia tiempo. Adaptada de Arciniega y Jaime (2011).

Siguiendo con la interpretación de resultados, en general los ítems relacionados con la gráfica de $v$ vs $t$ (del 13 al 22) tienen menores aciertos que los de la gráfica de $d$ vs $t$. En la gráfica de $d$ vs $t$, traducen correctamente el término velocidad constante, sin embargo en la gráfica de $v$ vs $t$, menos de la mitad de los alumnos que contestaron correctamente a la anterior, lo hacen (ver ítems 12 y 21 en la Figura 2).

Los ítems 16 y 17 para la gráfica de velocidad son los de menor frecuencia de aciertos, ambos items corresponden a la habilidad de los alumnos de explicitar los supuestos, esto ya ha sido discutido con los ítems 5 y 6 de la gráfica de $d$ vs $t$. 


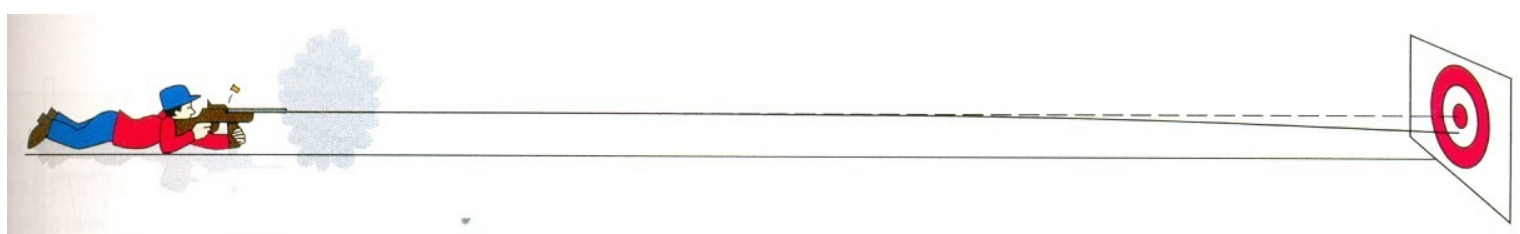

figura 3.17 Una tirador dispara a un blanco distante. La bala cae mientras viaja hacia el objetivo.

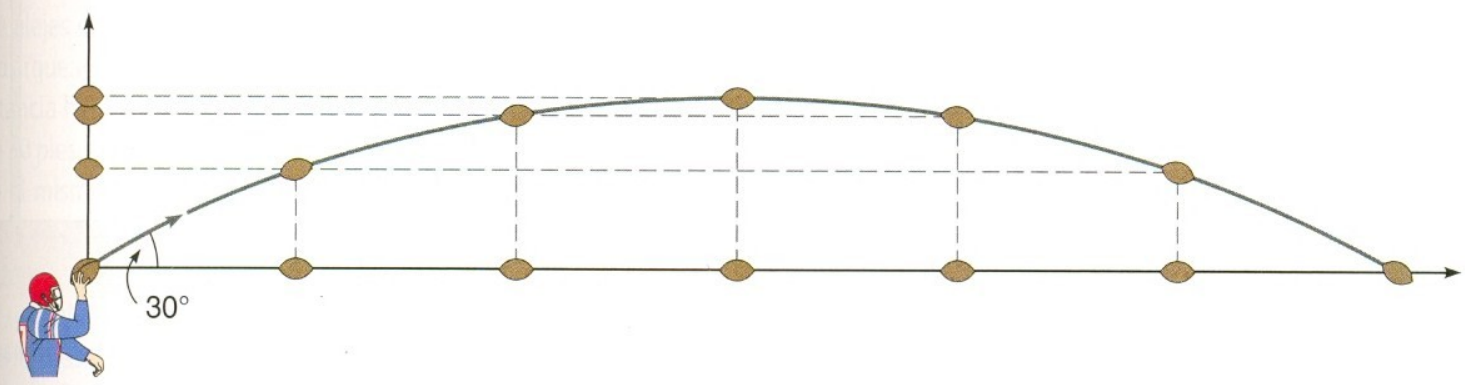

figura 3.18 El vuelo de un balón de futbol lanzado en un ángulo de $30^{\circ}$ respecto a la horizontal. Las posiciones vertical y horizontal del balón se muestran en intervalos de tiempo regulares.

Figura 4. Diagrama de distancia tiempo. Adaptada de Griffith (2008)

\section{Conclusiones}

De los resultados se puede concluir que una de las principales recurrencias es que los alumnos no identifican los supuestos al resolver un problema, al punto que los confunden con la información presentada en el mismo.

En el ejercicio existe mucha información que no se utiliza para hacer las gráficas y los alumnos no están habituados a seleccionar la información. Los ejercicios de libros y de clase contienen, generalmente, sólo la información necesaria para su resolución; en caso de haber información adicional, se presenta como preámbulo del problema y es claramente irrelevante para la resolución del mismo.

A partir de este trabajo concluimos que los alumnos carecen de entrenamiento para resolver ejercicios no estructurados donde son tres los puntos conflictivos:

1. Seleccionar la información relevante

2. Traducirla al lenguaje gráfico.

3. Hacer suposiciones.

También podemos decir que, parte de la dificultad en este ejercicio consistió en que las instrucciones vienen al final de la lectura, de tal forma que cuando los alumnos leen, no saben para qué están leyendo, además no quieren retroceder para revisar. Por el contrario, en los problemas estructurados, los datos y las preguntas están en el mismo bloque y, por omitir información innecesaria, son párrafos más breves.

Por último, se dan algunas recomendaciones didácticas:

Consideramos que practicar la traducción de lenguajes en el aula mejorará la comprensión del enunciado de los problemas, primer paso en la resolución de los mismos. Además el uso de ejercicios no estructurados permite el desarrollo de otras habilidades del pensamiento, necesarias en la resolución de problemas. 
Puesto que el aprendizaje no es una simple construcción, sino una compleja reconstrucción de significados (lo que implica identificar los obstáculos de aprendizaje) conviene recordar el papel de la experiencia del estudiante en la formación de preconceptos. No nos referimos exclusivamente a la experiencia cotidiana sino, sobre todo, a la de otros cursos y a la inducida por los libros de texto.

En el contexto escolar, es claro que se imponen las reglas y significados de la academia; son las que, en último término, darán la calificación al alumno. Conviene que los ejercicios de clase muestren explícitamente los alcances de las representaciones modificando los datos y condiciones del problema original.

Para la fábula analizada en este artículo se podrían, por ejemplo, haber hecho las siguientes modificaciones:

• ¿Cómo sería la gráfica si la liebre no descansa?

• ¿Cómo sería la gráfica si la liebre parte al mismo tiempo?

- ¿Si la tortuga parte después?

- ¿Cómo sería la gráfica si la liebre no descansa, corre más rápido que la tortuga y, sin embargo, pierde?

\section{Referencias bibliográficas}

Arciniega, G. A. y Jaime, L. G. (2011) Física 1, México: Fernández editores.

Esopo, (2011) Fábulas. Vol II. Primera edición, Buenos Aires.Tecnibook, Alsina ediciones.

Feldenkrais, M. (1981) La dificultad de ver lo obvio. Buenos Aires: Paidós.

Flores-Martínez, P. y Peñas-Troyano, M. (2003). Formación inicial de profesores de matemáticas reflexivos. Revista Educación y Pedagogía. Universidad de Antioquia, 35, 95116.

Gutiérrez-Rodilla, B. M. (1998) La ciencia empieza en la palabra. Barcelona: Ediciones Península.

Griffith, W. T. (2008) Física Conceptual. México: McGraw-Hill Interamericana.

National Council of Teachers of Mathematics. http://www.nctm.org/standards/content. aspx?id $=4294967312$.

Consultada 15/02/2012

Newton, I. (1707) Arithmetica Universalis; sive de Compositione et Resolutione Arithmetica. Ghent University.

Polya, G. (1989) Cómo plantear y resolver problemas. México: Edit. Trillas.

Rodríguez-Lagunas, J. (2009) La deserción escolar en la UAM-I. México: Universidad Autónoma Metropolitana.

Sanfelices Cuevas, L. (1990) La comprensión de lectura en textos de ciencias naturales. Enseñanza de las ciencias, 8(1), 59-64. 


\section{Anexo 1}

\section{Instrucciones:}

Lee la fábula siguiente y realiza las actividades que se encuentran en la siguiente hoja.

\section{La liebre y la tortuga}

Esopo

En el mundo de los animales vivía una liebre muy orgullosa, porque ante todos decía que era la más veloz: Por eso, constantemente se reía de la lenta tortuga.

-jMiren la tortuga! ¡Eh, tortuga, no corras tanto que te vas a cansar de ir tan de prisa! -decía la liebre riéndose de la tortuga.

Un dia, conversando entre ellas, a la tortuga se le ocurrió de pronto hacerle una rara apuesta a la liebre.

-Estoy segura de poder ganarte una carrera -le dijo.

- ¿A mi? -preguntó, asombrada, la liebre.

-Pues sí, a ti. Pongamos nuestra apuesta en aquella piedra y veamos quién gana la carrera.

La liebre, muy divertida, aceptó.

Todos los animales se reunieron para presenciar la carrera. Se señaló cuál iba a ser el camino y la llegada. Una vez estuvo listo, comenzó la carrera entre grandes aplausos.

Confiada en su ligereza, la liebre dejó partir a la tortuga y se quedó remoloneando. iV aya si le sobraba el tiempo para ganarle a tan lerda criatura!

Luego, empezó a correr, corría veloz como el viento mientras la tortuga iba despacio, pero, eso sí, sin parar. Enseguida, la liebre se adelantó muchísimo. Se detuvo al lado del camino y se sentó a descansar.

Cuando la tortuga pasó por su lado, la liebre aprovechó para burlarse de ella una vez. más. Le dejó ventaja y nuevamente emprendió su veloz. marcha. Varias veces repitió lo mismo, pero, a pesar de sus risas, la tortuga siguió caminando sin detenerse. Confiada en su velocidad, la liebre se tumbó bajo un árbol y abi se quedó dormida.

Mientras tanto, pasito a pasito, y tan ligero como pudo, la tortuga siguió su camino hasta llegar a la meta. Cuando la liebre se despertó, corrió con todas sus fuerzas pero ya era demasiado tarde, la tortuga babia ganado la carrera. 
Trimestre que cursas

Licenciatura

Cursé Mecánica y fluidos: Este trimestre

En trimestres anteriores

Nunca

\section{Actividades}

Considera:

- La meta está a 1500 metros del origen.

- La tortuga corrió con velocidad constante y tardó dos horas en llegar a la meta.

- La liebre se detuvo a descansar tres veces y corrió con velocidad constante.

1. Traza las gráficas, en la siguiente cuadrícula, de las distancias recorridas por la tortuga y la liebre como función del tiempo. Escribe las unidades correspondientes para cada eje. Si tienes que hacer suposiciones escríbelas.

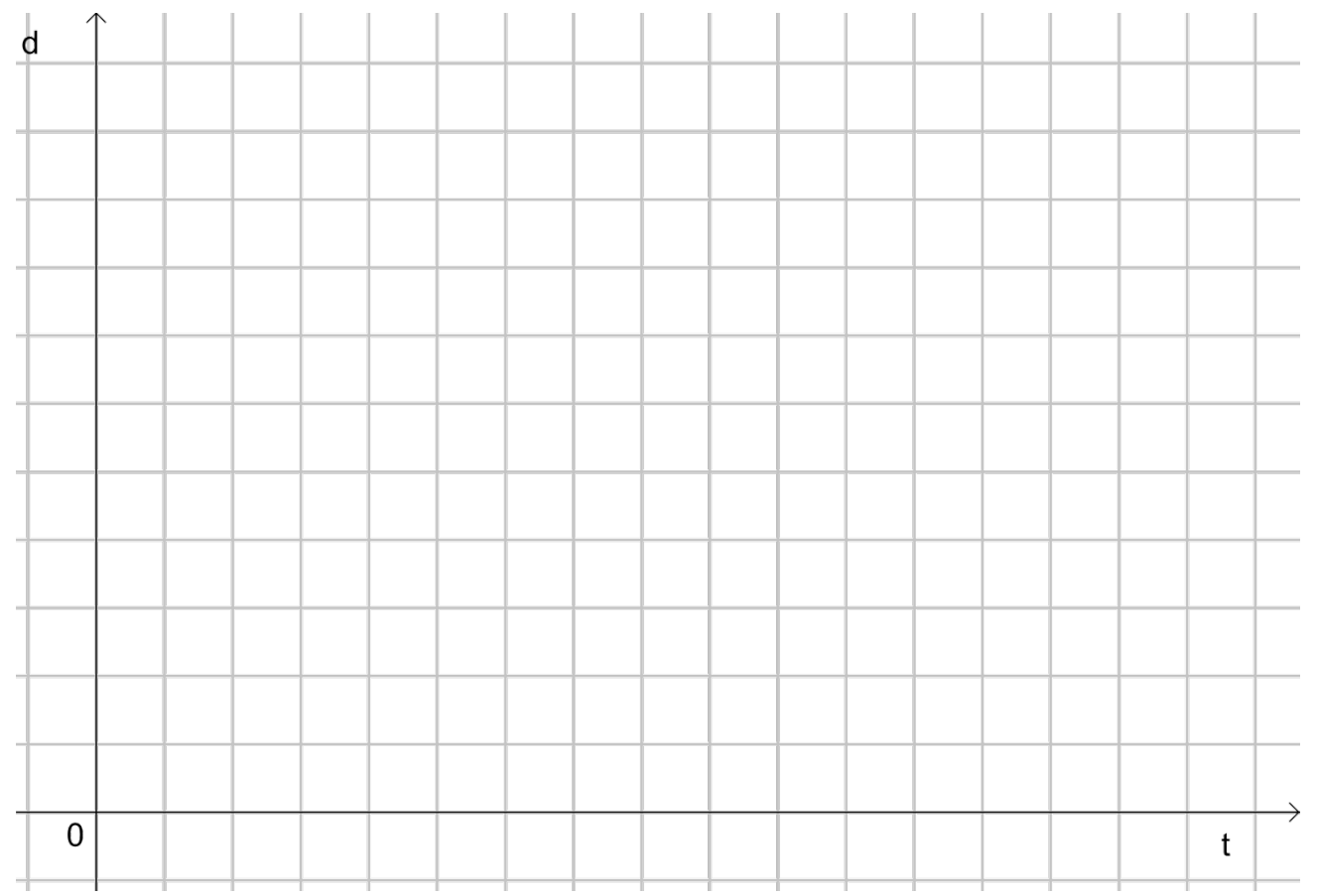

Escribe aquí tus suposiciones: 
2. Traza las gráficas, en la siguiente cuadrícula, de las velocidades de la tortuga y la liebre como función del tiempo. Escribe las unidades correspondientes para cada eje. Si tienes que hacer suposiciones escríbelas.

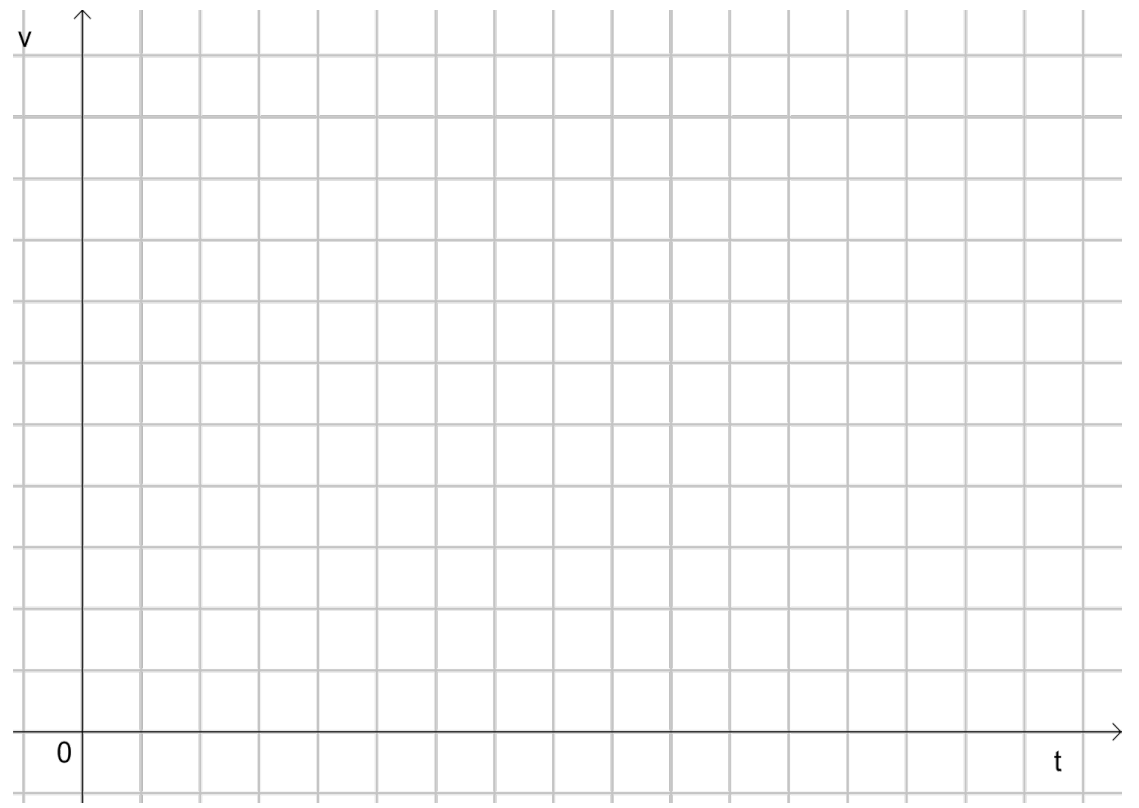

Escribe aquí tus suposiciones:

Lista de cotejo

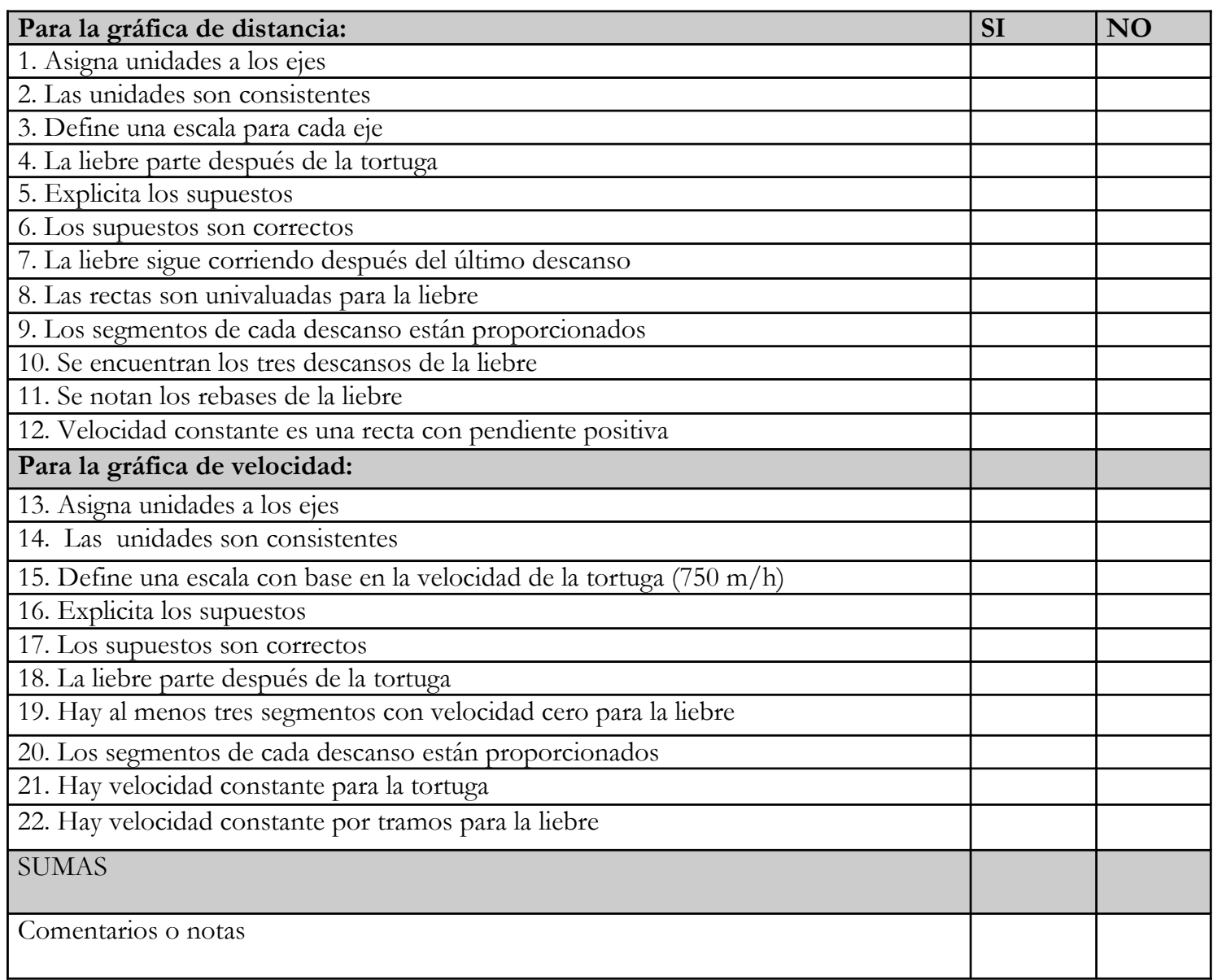

